\title{
Hadronic Equipartition of Quark and Glue Momenta
}

\author{
Y.N. Srivastava and A. Widom \\ Physics Department, Northeastern University, Boston MA 02115
}

\begin{abstract}
If the "glue" which binds quarks within hadrons takes the form of strings, then a virial theorem may be derived which shows how the total hadron four momentum splits up into a quark contribution plus a glue contribution. The hadrons made up of light quarks exhibit an equipartion of four momentum into equal parts quarks and glue. The agreement with the experimental "parton" distribution four momentum sum rule is quite satisfactory as is the string fragmentation model.

PACS: 14.65.Bt, 14.70.Dj, 12.39.Ki, 12.38.Aw
\end{abstract}

\section{INTRODUCTION}

The quark model presently plays a role in the classification of hadronic particles similar to the role played by the periodic table of chemical elements in the nineteenth century. From a microscopic viewpoint, the interaction holding the quarks together is often taken to be described by the Yang-Mills glue of quantum chromodynamics (QCD). However, the QCD derivation of the quark confinement within the hadron is more than just a little obscure. In order to make contact with experiments, some effective model of confinement must be constructed. The models include bags or string fragmentation constructions. The word "glue", within a given model, here denotes whatever the physical substance (perhaps Yang-Mills gauge gluons) that binds the quarks together.

Of the QCD inspired quark binding pictures, perhaps the most popular among experimentalists is the string fragmentation model [1] [2]. This model can be partly understood as a consequence of the Wilson closed loop conjecture [3]. Briefly, Wilson considered the action of sending a quark with QCD charge $g$ around a closed space-time path $\partial \Sigma$ which bounds a space-time area $\Sigma$; i.e the vacuum Wilson action $W$ is defined by

$$
\exp \left(\frac{i W}{\hbar}\right)=\left\langle 0\left|\exp \left(\frac{i g}{\hbar c} \int_{\partial \Sigma}\left(A_{\mu}^{c} \lambda_{c} / 2\right) d x^{\mu}\right)\right| 0\right\rangle_{+}
$$

where the subscript + denotes path ordering. The Wilson conjecture is that the action is proportional to the area for sufficiently large loops; i.e.

$$
W=(\tau / c) \Sigma
$$

In order to see the physical significance of the proportionality constant $\tau$, consider a rectangular space-time
Wilson loop as shown in Fig. 1. The particle moves forward in time $T$ as a quark, space-like displacement $R$ as a string, backward in time $-T$ as an anti-quark, and space-like displacement $-R$ as a string. The area of the loop is

$$
\Sigma=-c R T
$$

where the negative sign comes from the factor $(-1)$ in the Lorentz metric for time-like intervals. The Wilson loop action for this case is then given by

$$
W=-\tau R T \text {. }
$$

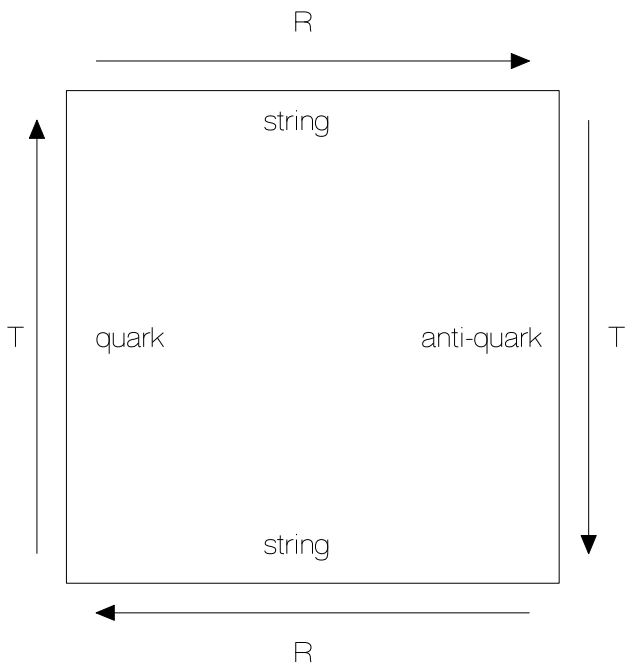

FIG. 1. In the above rectangular Wilson loop in space-time, the quark moves forward in time while the anti-quark moves backward in time. On the space-like top and bottom sides of the loop, the superluminal quark path forms a string. The oriented area enclosed by the rectangular loop is $\Sigma=-c R T$ and the action $W=(\tau / c) \Sigma$.

For an observer of the loop, the physical situation is as follows: There exists a quark and an anti-quark at fixed points in space connected by a string of length $R$. The string energy $E_{\text {string }}$ for this situation is contained in the action $W$ via the quantum mechanical rule for the time translation amplitude,

$$
\exp (i W / \hbar)=\exp \left(-i E_{\text {string }} T / \hbar\right) .
$$

The energy of the string of length $R$ is thereby 


$$
E_{\text {string }}=\tau R \text {. }
$$

The parameter $\tau$ in the Wilson loop Eq.(2) is merely the string tension.

If the Wilson conjecture holds true, then a meson which consists of a quark (at position $\mathbf{r}_{1}$ ) and an antiquark (at position $\mathbf{r}_{2}$ ) exhibits confinement from a "string potential energy"

$$
U_{\text {meson }}\left(\mathbf{r}_{1}, \mathbf{r}_{2}\right)=\tau\left|\mathbf{r}_{1}-\mathbf{r}_{2}\right| \text {. }
$$

More generally, if the action of Wilson loops scales as the area, then a potential energy for $n$ constituent quarks obeys the scaling law

$$
U\left(\xi \mathbf{r}_{1}, \ldots, \xi \mathbf{r}_{n}\right)=\xi^{h} U\left(\mathbf{r}_{1}, \ldots, \mathbf{r}_{n}\right)
$$

where

$$
h=1 \quad \text { (Wilson Strings). }
$$

The purpose of this work is to examine the consequences of homogeneous scaling potentials for the partitioning of the hadron rest energy $M c^{2}$ into quark energy and glue energy. If the hadron is made up of light quarks, then it turns out that Wilson scaling implies that the rest energy of the hadron is in equal parts quark energy and glue energy. This equipartitioning of hadron rest energy is in experimental agreement with strong interaction data, as will be discussed in Sec. IV. If the hadron contains heavy quarks, then Wilson scaling predicts deviations from the equipartition of energy rule.

In Sec. II, we consider the virial for an arbitrary number of quarks and derive a relativistic virial theorem which relates the total mean 4-momentum of the quarks to that of the glue and an spatial integral over the trace of the quark energy-momentum tensor. In Sec. III, we show that for a hadron made of light quarks (i.e., in the limit of vanishing quark masses) obeying Wilson scaling, the mean energy carried by the quarks is equal to that carried by the glue. In Sec. IV, we review briefly the well known data on the "parton" distributions in the best studied case of the proton confirming our theoretical results obtained in Sec. III. We close the paper with some concluding remarks in Sec. V.

\section{RELATIVISTIC VIRIAL THEOREM}

If the positions and momenta of the quarks are denoted by $\left(\mathbf{r}_{1}, \ldots \mathbf{r}_{n}, \mathbf{p}_{1}, \ldots \mathbf{p}_{n}\right)$ and if, in the center of mass frame, the positions and momenta of the quarks are bounded, then the virial

$$
\mathcal{V}=(1 / 2) \sum_{j=1}^{n}\left(\mathbf{r}_{j} \cdot \mathbf{p}_{j}+\mathbf{p}_{j} \cdot \mathbf{r}_{j}\right)
$$

is also bounded. The internal (center of mass frame) wave function $\Psi$ of a hadron having mass $M$ obeys

$$
\mathcal{H} \Psi=M c^{2} \Psi
$$

Hence,

$$
\langle\Psi, \dot{\mathcal{V}} \Psi\rangle=(i / \hbar)\langle\Psi,[\mathcal{H}, \mathcal{V}] \Psi\rangle=0
$$

The rate of change (in time) of the virial Eq.(10) obeys

$$
\langle\Psi, \dot{\mathcal{V}} \Psi\rangle=\left\langle\Psi, \sum_{j=1}^{n}\left(\mathbf{v}_{j} \cdot \mathbf{p}_{j}+\mathbf{r}_{j} \cdot \mathbf{f}_{j}\right) \Psi\right\rangle=0,
$$

where the velocity

$$
\mathbf{v}_{j}=(i / \hbar)\left[\mathcal{H}, \mathbf{r}_{j}\right]=\left(\partial H_{j} / \partial \mathbf{p}_{j}\right)=c \alpha_{j}
$$

is calculated from the Dirac Hamiltonian $H_{j}$ of the $j^{\text {th }}$ quark. If the quark mass is $m_{j}$, then

$$
H_{j}=c \alpha_{j} \cdot \mathbf{p}_{j}+m_{j} c^{2} \beta_{j} .
$$

The force on the $j^{\text {th }}$ quark

$$
\mathbf{f}_{j}=\dot{\mathbf{p}}_{j}=(i / \hbar)\left[\mathcal{H}, \mathbf{p}_{j}\right]
$$

is calculated from a potential of the binding glue

$$
\mathbf{f}_{j}=-\operatorname{grad}_{j} U
$$

The potential obeys the Wilson scaling Eqs.(8) and (9).

From the nature of the Dirac Hamiltonian Eq.(15), it follows that

$$
\sum_{j=1}^{n} \mathbf{p}_{j} \cdot \mathbf{v}_{j}+\sum_{j=1}^{n} m_{j}\left(\partial H_{j} / \partial m_{j}\right)=\sum_{j=1}^{n} H_{j}
$$

while the Euler equation corresponding to Eq.(8) is well known to be

$$
\sum_{j=1}^{n} \mathbf{r}_{j} \cdot \mathbf{f}_{j}=-\sum_{j=1}^{n} \mathbf{r}_{j} \cdot \operatorname{grad}_{j} U=-h U .
$$

The mean energy of the quarks,

$$
\bar{E}_{\text {quarks }}=\left\langle\Psi, \sum_{j=1}^{n} H_{j} \Psi\right\rangle
$$

and the mean energy of the glue,

$$
\bar{E}_{\text {glue }}=\langle\Psi, U \Psi\rangle,
$$

are related via Eqs.(13) and (18-21) as in the following

\section{Virial Theorem:}

$$
\bar{E}_{\text {quarks }}=h \bar{E}_{\text {glue }}+\left\langle\Psi, \sum_{j=1}^{n} m_{j}\left(\partial H_{j} / \partial m_{j}\right) \Psi\right\rangle \text {. }
$$

The second term on the right hand side of Eq.(22) is related to the trace of the quark contribution $T^{\mu \nu}$ to the mean local hadron stress-energy tensor; i.e. 


$$
-\int_{\text {hadron }} T_{\mu}^{\mu}(\mathbf{r}) d^{3} \mathbf{r}=\left\langle\Psi, \sum_{j=1}^{n} m_{j}\left(\partial H_{j} / \partial m_{j}\right) \Psi\right\rangle .
$$

The trace of the stress-energy tensor is simply expressed in terms of the local quark energy density $\varepsilon$ and the local quark pressure $P$; i.e.

$$
-T_{\mu}^{\mu}(\mathbf{r})=\varepsilon(\mathbf{r})-3 P(\mathbf{r}) .
$$

Thus, the virial theorem follows from Eqs.(22-24) to read as

$$
\bar{E}_{\text {quarks }}=h \bar{E}_{\text {glue }}+\int_{\text {hadron }}\{\varepsilon(\mathbf{r})-3 P(\mathbf{r})\} d^{3} \mathbf{r} .
$$

Finally, let us here relax the restriction of working in the hadron center of mass frame. In terms of the total four momentum of the hadron $\left(P^{\mu}=\bar{P}_{\text {quarks }}^{\mu}+\bar{P}_{\text {glue }}^{\mu}\right)$,

$$
\bar{P}_{\text {quarks }}^{\mu}=h \bar{P}_{\text {glue }}^{\mu}-\left(\frac{1}{c}\right) \int_{\text {hadron }} T_{\lambda}^{\lambda}(x) d^{3} \sigma^{\mu}(x),
$$

where the four vector " 3 -volume components" are defined via

$$
d^{3} \sigma_{\mu}(x)=\left(\frac{1}{3 !}\right) \epsilon_{\mu \nu \lambda \beta} d x^{\nu} \wedge d x^{\lambda} \wedge d x^{\beta} .
$$

The above manifestly Lorentz invariant form of the virial theorem Eq.(26) is the central result of this section.

\section{LIGHT QUARK EQUIPARTITION OF HADRON ENERGIES}

For hadrons made up of light quarks, one expects the "ultra-relativistic" quark gas result $\varepsilon \approx 3 P$. The precise theorem is that in the limit of vanishing quark masses

$$
-\lim _{m \rightarrow 0} T_{\mu}^{\mu}=\lim _{m \rightarrow 0}(\epsilon-3 P)=0 .
$$

Eqs.(9), (25) and (28) imply the equipartition of the hadron rest energy $M c^{2}=\bar{E}_{\text {quark }}+\bar{E}_{\text {glue }}$ in Wilson loop string models; i.e.

$$
\bar{E}_{\text {quark }}=\bar{E}_{\text {glue }} \quad \text { (light quarks on strings). }
$$

To see simply how Eq.(29) arises, let us consider a simple model of mesons made up of a quark and an antiquark and let us employ the string potential of Eq.(7); $U_{\text {meson }}(R)=\tau R$ where $R$ is the length of the string. The ultra-relativistic quark kinetic energy corresponding to a meson angular momentum $J$ is given by $K_{\text {meson }}(R)=$ $2 c(J / R)$. The mass of the meson may be estimated by the minimum energy principle [4 6 .

$$
M_{J} c^{2}=\inf _{0<R<\infty}\left\{K_{\text {meson }}(R)+U_{\text {meson }}(R)\right\} .
$$

The physical string length $R_{J}$ then obeys

$$
R_{J}^{2}=(2 c J / \tau),
$$

while the mass squared determines a linear angular momentum trajectory

$$
M_{J}^{2}=\left(8 \tau J / c^{3}\right) .
$$

For the above (perhaps overly) simple model, note that $\bar{E}_{\text {quark }}=K_{\text {meson }}\left(R_{J}\right)=U_{\text {meson }}\left(R_{J}\right)=\bar{E}_{\text {glue }}$. Thus, Eq.(29) holds true.

However, the equipartition of energy in Eq.(29) is not a trivial physics result. Different models yield different partitions of the energy. For some "bag" models, [7] $U_{\text {meson }}(R ; \mathrm{bag}) \approx(4 \pi / 3) B R^{3}$, or more generally

$$
h \approx 3 \quad \text { (bag models). }
$$

Applying the virial theorem for this case yields

$$
\bar{E}_{\text {quark }} \approx 3 \bar{E}_{\text {glue }} \quad \text { (light quarks in bags). }
$$

On the basis of Eqs.(29) and (34) and the discussion in Sec. IV, it appears that string models are experimentally favored over bag models.

\section{IV. "PARTON" DISTRIBUTION DATA}

Our results from Secs. II and III are that for light quark bound states such as the proton and the pion, we expect the $50 \div 50$ rule for the momenta carried by the quarks and the glue. Here we shall briefly discuss an experimental verification of this fact for the proton in the context of the parton model. As well known (see for example [8] [9]), one defines a quark density $q(x)$, and antiquark density $\bar{q}(x)$ and a gluon density $G(x)$, where $x$ is the fraction of the proton momentum. What has been found experimentally is that

$$
\sum_{q} \int_{o}^{1}(d x)[q(x)+\bar{q}(x)] \approx 0.5 .
$$

To quote ref.[9], "[the above] is an experimental result. It indicates that the quarks carry about $50 \%$ of the proton's momentum. The rest is attributed to gluon constituents."

There are similar albeit less accurate data for the pion structure function as well showing the equipartition of the pion momenta into quarks and glue.

We expect sizeable deviations from the equipartition for large quark masses. Present data for heavy quarks are not sufficiently accurate to allow for a quantitative test.

\section{CONCLUSIONS}

In the preceding, we have derived a relativistic virial theorem valid for a generic hadron. For homogeneously 
scaling potentials, it connects the mean energy carried by the quarks, the glue and the trace of quark energymomentum tensor integrated over the hadronic volume. For "ordinary" hadrons such as the pion and the nucleon, made of light quarks, it leads to the pleasing result that for the string model (which satisfies the Wilson area conjecture) the mean quark energy equals the mean energy carried by the glue. There is ample experimental evidence via the quark and gluon densities verifying this $50 \div 50$ rule for the case of the proton. (A less precise but positive confirmation is also found experimentally for the pion). The "bag models" generally fail in this respect.

[1] R. P. Feynman, "Photon Hadron Interactions", W. A. Benjamin, New York (1972).

[2] J. D. Bjorken, Phys. Rev. 179 (1969) 1547.

[3] K. Wilson, Phys. Rev D10 (1974) 2445.

[4] S. Filipponi, G. Pancheri and Y. Srivastava, Phys. Rev. Lett. 80 (1998) 1838; ibid 82 (1999) 458.

[5] S. Filipponi, G. Pancheri and Y. Srivastava, Phys. Rev. D58 (1999) 76003.

[6] G. Pancheri, Y. Srivastava and A. Widom, "Hadronic masses and widths through Regge trajectories", Invited talk by YS at of the Heavy Flavour Workshop held at Frascati, March 1999. Proceedings published in Frascati Physics Series, Editors T. Bressani, A. Feliciello and A. Filippi.

[7] J. Donoghue, B. Holstein and E. Golowich, "Dynamics of the standard model", Cambridge University Press, New York (1995).

[8] V. Barger and R. J. Phillips, "Collider Physics", Addison Wesley Publishing Co., California (1987).

[9] W. K. Ellis, W. J. Stirling and W. R. Webber, "QCD and Collider Physics", Cambridge University Press, New York (1996). 